\title{
Civilisations
}

Revue internationale d'anthropologie et de sciences

humaines

$41 \mid 1993$

Mélanges Pierre Salmon II

\section{Aux origines du royaume de Ngoyo}

\section{Habi Buganza Mulinda}

URL : http://journals.openedition.org/civilisations/1702

DOI : 10.4000/civilisations. 1702

ISSN : 2032-0442

\section{Éditeur}

Institut de sociologie de l'Université Libre de Bruxelles

\section{Édition imprimée}

Date de publication : 1 septembre 1993

Pagination : 165-187

ISBN : 2-87263-094-5

ISSN : 0009-8140

\section{Référence électronique}

Habi Buganza Mulinda, "Aux origines du royaume de Ngoyo », Civilisations [En ligne], 41 | 1993, mis en ligne le 29 juillet 2009, consulté le 23 avril 2019. URL : http://journals.openedition.org/ civilisations/1702 ; DOI : 10.4000/civilisations.1702

Ce document a été généré automatiquement le 23 avril 2019

(c) Tous droits réservés 


\title{
Aux origines du royaume de Ngoyo
}

\author{
Habi Buganza Mulinda
}

\section{Introduction}

1 Il est significatif de constater qu'alors qu'en Afrique, et particulièrement au Zaïre, au moment de la commémoration de l'arrivée de Diégo Câo à l'embouchure du fleuve Zaïre, il ne fut question que de cinq siècles d'évangélisation, en Europe, la "Découverte de l'Amérique" soit l'objet de vastes célébrations (l'Exposition de Séville) et que toute l'année est pleine de rencontres scientifiques sur ce même thème. Tout se passe comme si le christianisme fut le seul lien entre l'Europe et l'Afrique et comme si personne ne voulait se rappeler de tant de souvenirs malheureux qui ont abouti aux actuelles relations inégales.

Quoi qu'il en soit, nous n'avons aucunement l'intention d'approfondir ce problème. Notre objectif est d'évoquer les circonstances diverses qui ont permis au petit état côtier de Ngoyo de se former et de s'émanciper de la tutelle de ses voisins vers la fin du $16^{\mathrm{e}}$ siècle. Nous passerons en revue les éléments internes et externes qui ont favorisé l'émergence de cet Etat ainsi que les conditions qui lui permirent d'être un moment le partenaire des commerçants Portugais et Hollandais; situation qui ne dura pas puisque la traite atlantique des esclaves transforma rapidement ses dirigeants en simples courtiers.

Pour ce faire, nous avons utilisé la nombreuse littérature existant sur les Etats de la Côte occidentale de l'Afrique Centrale. Une lecture critique permet d'en tirer de nombreux renseignements s'étendant sur près de cinq siècles. Ces éléments ont été enrichis de quelques données de la tradition orale, qui, pensons-nous, malgré leur aspect lacunaire, peuvent éclairer l'histoire de cette région. Ces données ont été récoltées sur le terrain lors des missions effectuées chez les Woyo habitant le territoire connu au Zaïre sous la dénomination administrative de "Collectivité de la Mer". 


\section{L'origine des Woyo}

4 Il n'est pas nécessaire de remonter jusqu'à l'Egypte des Pharaons comme essaie de le faire Nguvulu ${ }^{1}$ pour prouver l'ancienneté des Woyo sur le territoire qui fut le royaume de Ngoyo. Des indices comme l'archaïsme linguistique et quelques éléments des fouilles archéologiques ${ }^{2}$ montrent que ce territoire au nord du fleuve Zaïre fut habité depuis longtemps. Par contre, comme pour plusieurs peuples de l'Afrique centrale, il est difficile de déterminer avec exactitude l'origine des Woyo et, pour exposer le mouvement migratoire des peuples de cette région ${ }^{3}$, il faut souvent se contenter des hypothèses ébauchées par les historiens. Pour l'ensemble, ce mouvement s'oriente du nord au sud, mais il n'exclut pas des migrations ultérieures en sens contraire : c'est le cas de ce que dit Hagenbucher-Sacripanti, qui, s'agissant des traditions Vili (Loango), rapporte que des Woyo connus sous le nom de Bavanji, auraient été les premiers à arriver à Loango et seraient à l'origine du royaume de Loango $0^{4}$.

5 Les traditions Woyo elles-mêmes sont rarement explicites à ce sujet. Néanmoins, la légende de Buunzi qui explique l'origine du pouvoir, situe le point de départ du groupe qui accompagna cette mère fondatrice à Kikungulu, au nord de Ngoyo, au royaume de Loango.

6 Carlos M.H. Serrano ${ }^{5}$ qui a essayé d'utiliser les traditions des grandes familles de Ngoyo, n'aboutit pas à des résultats plus heureux: pour certaines, il remonte jusqu'au $17^{\mathrm{e} m e}$ siècle, mais le panorama qui se dégage est celui de la fin du $18^{\mathrm{e}}$ siècle et du début du $19^{\mathrm{e}}$ siècle.

7 En fait, cette faiblesse de profondeur des traditions indique que la population de Ngoyo est d'origines diverses. De par sa position géographique, au nord du fleuve Zaïre, on peut constater que le territoire de Ngoyo fut un lieu de passage important des migrations venant du nord vers le sud et vice-versa. C'est ainsi que, comme le rapporte la légende de Buunzi, plusieurs informateurs confirment l'apport humain des Vili dans la composition de la population Woyo. De plus, il existait en permanence à l'insida, Sanctuaire de l'Esprit de la terre Buunzi situé non loin de Muanda, une colonie de Vili dont le quartier situé au Nord-est était connu sous l'appellation de Belo cia Bavili (quartier des Vili) ${ }^{6}$ et que périodiquement des pélerins Vili y séjournaient ${ }^{7}$. Plus au sud du royaume de Ngoyo, par contre, des Salongo et des Kongo ont traversé le fleuve et se sont installés sur le territoire des Woyo. C'est le cas de plusieurs chefs qui, au siècle passé, dirigèrent la principauté de Muanda mais aussi de plusieurs familles habitant les villages zaïrois à l'est de la Collectivité de la Mer. D'autres habitants sont certainement les descendants de nombreux immigrés qui y ont été attirés par les richesses produites par ce royaume. En effet, la mer et les rivières sont poissonneuses et plusieurs salines produisaient du sel que venaient chercher par caravanes des personnes originaires de régions parfois très éloignées. De plus, Dapper nous apprend que les Woyo produisaient des tissus en raphia très recherchés dont ils avaient le quasi monopole ${ }^{8}$. Quand on sait que certains de ces tissus servaient de monnaie (mbongo), même dans des contrées lointaines (Kwango par exemple), on peut s'imaginer que le territoire attira les étrangers.

8 Pour les visiteurs européens, les Woyo diffèrent peu de leurs voisins du Kakongo et du Loango. Les langues et les moeurs sont semblables ${ }^{9}$. Certains traits de caractère leur sont cependant spécifiques. Ils sont connus pour leur agressivité envers leurs voisins et les étrangers. Ainsi, alors que Dapper rapporte qu'ils avaient la réputation d'être des bons 
soldats ${ }^{10}$, John Olgiby les traite d'impudiques en disant que "yet towards strangers, they are churlish and uncivil"11. Plusieurs missionnaires rapportent le grand attachement des Woyo à leur religion traditionnelle.

9 Merolla parle de peuple qui s'adonne à la sorcellerie et à la magie ${ }^{12}$ avec ce que ces mots avaient comme charge négative et de méprisant à l'époque; il souligne aussi l'inimitié qu'ils ont contre la religion chrétienne et ceux qui la pratiquent ${ }^{13}$.

10 Plus tard, les Woyo (appelés souvent Kabindes) seront utilisés dans la traite des esclaves, ils étaient considérés comme bons caravaniers : armés jusqu'aux dents, ils étaient réputés pour leur peu de pitié. Ils étaient aussi considérés comme bons gardiens de barracons, là où étaient entassés les esclaves avant l'embarquement. Ils étaient enfin connus pour leur capacité à s'expatrier comme matelots ou pilotes à bord des navires de guerre ou de commerce ${ }^{14}$. Sur terre, les Woyo suivaient les Portugais dans les petites villes créées le long des principales routes d'Angola où ils étaient souvent boys-lavandiers. Ils vivaient à part et avaient des fétiches ${ }^{15}$ de grande réputation.

\section{Création du Royaume de Ngoyo}

11 C'est probablement à la fin du $15^{\mathrm{e}}$ siècle que se forma sur le territoire des Woyo le royaume qui plus tard fut connu sous les appelations de royaume de Goy, Ngoy, Engoy, N'goie et N'goyo.

12 L'anglais Andrew Battel, qui arriva à Cabinda vers 1589, signale que Engoy se trouve au nord du fleuve Zaïre ${ }^{16}$ tandis que John Ogilby précise que "Goy borders in the shore on the south at the river Zaïre, or Congo, upon Kakongo on the south". Il ajoute que son principal port, Cabinda, se trouve à 6 heures de marche de Punto de Palmeiro ${ }^{17}$, sur le fleuve ${ }^{18}$. Dapper complète ces informations : "Ce royaume a la mer au couchant, le fleuve (Zaïre) au midi et les terres de Kakongo au levant et au septentrion ${ }^{19 " .}$ Par ailleurs, le missionnaire Merolla, qui fit un court séjour à Mbanza Ngoyo, la capitale, nous apprend que le royaume de Ngoyo était tellement petit qu'il n'avait de royaume que le nom ${ }^{20}$. Ce qui confirment les informations de l'abbé Proyart qui parle du "petit royaume de N'goyo" ${ }^{21}$. Les limites de Ngoyo ne vont pratiquement pas varier: le royaume se situe entre le $5^{\mathrm{e}}$ et $6^{e}$ de latitude sud. Il s'étend le long du littoral du nord au port de Malemba, au sud à la province de Manputu dont une partie fut concédée aux Solongo en 1631. Au nord, sa frontière est constituée par la rivière Lulonda et à l'est par les rivières Lukala et Kalamu ${ }^{22}$.

\section{Récits de fondation du Royaume}

13 Les traditions Woyo expliquent la fondation du royaume de Ngoyo par deux légendes articulées autour de deux personnages féminins. La première met en scène une femme du nom de Mpuenya qui serait venue du royaume de Kongo d'où elle aurait été chassée pour avoir donné secrètement naissance à des triplés, deux garçons Tumba et Lilu et une fille Silu ${ }^{23}$.

14 Avec ses enfants, elle serait parvenue à fuir et aurait débarqué au royaume de Ngoyo à un lieu appelé Vumu. Elle aurait séjourné à Fumba ${ }^{24}$ avant d'atteindre Mbanza Ngoyo où elle se maria à un noble Woyo et donna le jour à deux autres enfants, Panzu et Pukuta. Devenus grands, les enfants furent nommés chefs des territoires au nord du fleuve Zaïre : à Tumba échut le territoire de Loango, à Lilu, le Kakongo et à Silu, le Ngoyo. Le jour de son 
intronisation, cette dernière ne put se présenter en public : elle ne pouvait pas en effet sortir de la maison suivant ce que la tradition assigne aux femmes en règles menstruelles. Aussi ce fut son demi-frère Panzu qui fut investi du pouvoir au Ngoyo.

A l'inverse de ce récit, le second courant de la tradition Woyo ${ }^{25}$ attribue le rôle important de la fondation du royaume à une femme nommée Nguli ivwa mabeene, la mère-auxneufs-seins, ancêtre éponyme commune à Loango, Kakongo et Ngoyo. Postérieurement à la création de ces trois royaumes, elle serait aussi à l'origine du royaume de Kongo. Dans cette tradition, cette femme est assimilée à Cikulu, une princesse Vili qui entreprit un périple vers le sud en quête de terres et d'un sanctuaire. Dans ses pérégrinations à la tête d'un groupe de migrants, elle séjourna successivement au nord de la rivière Lukola au Kabinda et à Muanda (appelé aussi Muanda Mazi) et elle resta quelques temps dans l'île de Katala à l'embouchure du fleuve Zaïre. A Katala, elle devint medium de l'esprit Buunzi et passa par une expérience religieuse au bout de laquelle l'esprit Buunzi, qu'elle incarna désormais, l'enjoignit de quitter l'île de Katala et de s'installer à Cinsinda près de Muanda. Avec sa suite, elle passa par Vumu, localité située près de Banana, et elle prit la route de Cinsinda. C'est elle qui aurait réparti le pouvoir à Muanda, entre les responsables religieux, les responsables fonciers et les responsables politiques ${ }^{26}$. Ces derniers devaient "couvrir" (fuka) les premiers surtout en présence des étrangers. A cet effet, ils furent placés près de la mer, à Muanda Mazi, qui, pour cette raison, fut appelée aussi Muanda Sialuve (Muanda: terre de la palabre, de la justice politique). Les autres responsables suivaient, les religieux résidant à Cisinda autour d'un sanctuaire dédié à Buuzi. D'où le dicton "Cinsinda Wasinda Buunzi muna Katala", "Cinsinda qui fixa (arrêta) Buunzi venant de Katala". Là, Cikulu vécut une vie de recluse ; elle fut honorée comme Buunzi, principal nkisi si (esprit de terre), de la région. Avant sa mort, elle répartit les terres au nord du fleuve Zaïre entre ses descendants, notamment Loango, Kakongo et Ngoyo qui furent attribués à ses propres triplés, Maloango, Makongo et Mangoyo.

Il va sans dire que nous avons affaire ici à deux formes de légitimation du pouvoir royal qui prennent leurs points d'appui sur des éléments communs. Car, si l'on comprend que les deux récits fassent référence à deux personnages féminins, pour l'origine du pouvoir, compte tenu du système matrilinéaire de la société Woyo, on ne peut que s'interroger lorsque l'on considère les origines respectives de ces deux femmes, à savoir le royaume Kongo au sud et le royaume de Loango au nord. Sans vouloir en faire une analyse détaillée, relevons le fait qu'il s'agit de deux personnages en rupture avec le passé et à la recherche d'un avenir. Par ailleurs, les héritiers du pouvoir sont des triplés car il s'agit d'un chiffre valorisé dans la culture Woyo: le chiffre trois signifie la perfection. La naissance gémellaire est un signe du sacré et montre l'intimité des relations entre les dirigeants des trois royaumes, Loango, Kakongo et Ngoyo. Le passage à Vumu que l'on retrouve dans les deux récits souligne l'importance du sel comme produit de commerce et comme facteur important dans l'extension du pouvoir. Parmi les points de divergence, relevons l'origine des deux femmes fondatrices : l'une, Mpuenya, viendrait du royaume Kongo et serait donc la transition entre ce dernier et ceux du nord du fleuve ; l'autre viendrait de Loango, et serait porteuse à la fois du pouvoir et d'un message religieux, indépendants du royaume de Kongo. Il est probable que ces deux récits se réfèrent à deux moments différents de l'Histoire de Ngoyo : l'un marqué par l'influence Kongo, comme le suggèrent certains auteurs, l'autre caractérisé par la domination de Loango sur le nord du fleuve Zaïre comme le supposent les historiens et certaines traces dans la tradition. 


\section{Emergence du Royaume de Ngoyo}

17 L'émergence de Ngoyo comme entité politique autonome s'inscrit dans un processus historique général qui, au $14^{\mathrm{e}}$ siècle pour les uns, au $15^{\mathrm{e}}$ pour les autres, aboutit à l'organisation d'états centralisés en Afrique centrale. En fait, ce processus a bénéficié du concours de plusieurs facteurs relevant de la dynamique interne et/ou des circonstances extérieures.

Dans la dynamique interne, il faut prendre en considération les structures liées à l'organisation du territoire, celles qui ont trait à l'organisation de la société et celles qui favorisent les relations sociales.

Plusieurs auteurs ${ }^{27}$ attestent que le culte des esprits de la terre (bakisi basi) étaient en l'honneur chez les Woyo. Or l'organisation de ce culte est basée sur une division spatiale bien précise, à savoir une unité politico-religieuse ayant à sa tête dans les meilleures des cas un collège de responsables religieux, politique et foncier. Mais le plus souvent, le responsable religieux est considéré comme le représentant du clan propriétaire du sol et laisse l'exercice du culte à un délégué appelé ntoma si. Il est probable que le prestige de certains sanctuaires entraîna la prééminence de certains chefs politiques sur d'autres ( fummu nkazi, litt. chef du siège, kala = être assis). Ceci est d'autant plus plausible qu'on connaît l'importance du prêtre de Buunzi dans la désignation du roi du Loango ${ }^{28}$ ou de celui de Luunga dans le sacre du Mangoyo ${ }^{29}$.

Dans cet affermissement de l'autorité politique tendant à la formation du royaume de Ngoyo, on ne peut négliger le rôle important joué par les mécanismes sociaux. Ceux-ci assurent à l'organisation politique basée sur l'occupation spatiale, un équilibre et une cohérence internes ${ }^{30}$.

21 L'organisation sociale chez les Woyo, bien que basée sur la descendance matrilinéaire, tient compte des parents (buutu) tant paternels (bataata) que maternels (bamaama). Cependant, pour un individu, les rapports les plus significatifs ont lieu avec les parents maternels; d'abord dans le cadre d'un groupe restreint appelé nzo (littéralement maison) ou lumbu (enclos) comprenant les descendants d'une même aieule regroupés autour de l'oncle vivant le plus âgé (ngwa nkhazi) ${ }^{31}$ avec ses frères et ses soeurs. Regroupant deux générations, le nzo constitue une unité résidentielle, à l'intérieur d'un espace d'habitation qui est le village auquel chaque membre se réfère. Dans le cadre de la parenté cependant, l'unité est le vumu, ventre, lignage (ou vumu cia nguli, littéralement ventre de la femme). Il est théoriquement dirigé par l'homme le plus ancien (nkhazi) ${ }^{31}$. Le vumu est rarement localisé; il est généralement dispersé dans plusieurs villages suivant le gré des mariages des femmes membres du vumu.

Les vumu forment un groupe clanique (kaanda) sous l'autorité morale d'un chef de clan (ngwa nkanzi kaanda, ou fumu kaanda). Celui-ci est considéré comme le chef du plus ancien lignage issu de la fille aînée de l'ancêtre féminin éponyme. Au fur et à mesure de la centralisation de l'autorité du pouvoir politique, il semble que le rôle et l'autorité du chef de clan (ngwa khazi kaanda) se soient renforcé à la fois pour protéger les membres du clan contre les éventuelles exactions politiques et pour les représenter auprès des diverses hiérarchies politiques. Pour cela, le chef de clan devait être reconnu comme l'autorité suprême du clan. En effet, le clan étant un ensemble fluide du fait de sa dispersion sur le territoire, il fut nécessaire de recourir à une institution pouvant réunir ses membres autour d'un seul responsable du clan. 
(fumu kaanda) lors d'une cérémonie (kwalika bingu tshikaanda) où sont présents tous les matrilignages. Le chef ainsi investi porte le nom de nkundi mbaci et est responsable des trésors du clan (taa tshikanda) ${ }^{32}$. Il a pour rôle d'intercéder auprès des ancêtres par des libations quotidiennes aux autels familiaux (mayowa, li). Pour parvenir à bien assurer la gestion des membres du clan, il dispose d'un nkobe (reliquaire cylindrique) où sont placés des corbeilles contenant des objets représentant les puissances mystiques (bakisi) du clan. Ces forces sont généralement liées à la fécondité et à la prospérité du clan. Chaque année, ce chef de clan devait s'approprier ces forces lors d'un rituel (bingu) de culte aux ancêtres (nettoyage des cimetières) et de séances de réconciliation collective et de solidarité (repas collectifs après confessions publiques) ${ }^{32}$. Il est probable qu'à l'éclosion de la monarchie, les fumu kaanda, par ailleurs propriétaires de grands espaces territoriaux, formèrent un conseil d'où sortirent les premiers rois de Ngoyo. Ce sont des groupes claniques semblables, enrichis de diverses immigrations, qui ont fourni les rois à Ngoyo $^{33}$ et le roi, considéré comme chef suprême des chefs des clans, présidait un conseil des chefs de clan, tous investis dans le bingu ${ }^{34}$. Soulignons cependant qu'après avoir favorisé l'éclosion du royaume, ces grandes familles, dont les intérêts ont petit à petit divergé avec ceux de certains responsables du royaume, furent aussi à l'origine de la chute inéluctable de Ngoyo.

elles regroupent les personnes qui ont connu la même expérience de la maladie. On peut citer notamment le lemba, le mpanzu, ou le makwangu. De ces trois associations, le lemba semble être la plus ancienne. On constate en effet que le mpanzu comprend la guérison de la maladie du sommeil (apparue le siècle passé) et que le makwangu, un rituel féminin de possession, inclut des personnages portugais. Le lemba (du verbe lemba, calmer), chez les Woyo, est un rituel de guérison des maladies causées par le non respect des engagements de fidélité par un conjoint. En effet bien que n'impliquant pas un mariage monogamique, le lemba impose un engagement à une femme rituelle (généralement la première). L'association avait aussi comme spécialité les maladies se rapportant à la fécondité (impuissance, stérilité, avortements, etc.); elle était hiérarchisée et avait au sommet le grade de nkukunyungu, dont l'épouse était appelée nkam'ilemba (l'épouse dans le lemba) ${ }^{37}$.

Le rôle de ces associations dans le renforcement du royaume de Ngoyo ne provenait pas seulement des fonctions sociales qu'elles exerçaient mais des contacts qu'elles impliquaient entre les personnes. Elles permettaient à certains de bénéficier de la 
production, de l'accumulation et de la distribution des biens (produits palmistes et de pêche, sel, etc.).

Par ailleurs, les responsables occupaient des positions sociales qui leur permettaient la concentration des biens, notamment des biens de prestige comme les parures en métal ou les produits de la traite, biens qu'il fallait chercher loin, et exigeaient le contrôle du trafic et du marché.

On peut ajouter un autre facteur qui favorisa la formation et l'évolution du royaume de Ngoyo, à savoir la présence portugaise à la Côte Atlantique ${ }^{38}$. Il est curieux de constater en effet que l'émergence des royaumes au nord du fleuve Zaïre coïncide avec la présence de plus en plus grande des Portugais au sud du fleuve, c'est-à-dire au royaume de Kongo. Les Portugais voulaient faire de ce pays non seulement un modèle de christianisation, mais aussi un modèle d'organisation calquée sur celle du Portugal. Cette situation créa des contradictions à l'intérieur du royaume Kongo qui aboutirent du reste à un conflit armé en 1665. Cela permit progressivement aux royaumes du nord du fleuve de se libérer. Et même si le roi Avaro $1^{\mathrm{er}}$ de Kongo, vers 1583, clame, dans une lettre au Saint-Siège, sa souveraineté sur "Cakongo" et "Guyo", et qu'un document de 1595 décrivant la situation du royaume Kongo, cite Loango à la frontière Nord, on sait que vers $1535^{39}$, Loango est indépendant de Kongo. Vers 1570 du reste, le roi de Loango (Maloango) est un ami de celui de Kongo et il lui demande de l'aide contre les Teke ${ }^{40}$.

Vers 1589, d'après Battel, Ngoyo est encore dépendant de Loango ${ }^{41}$, mais petit à petit il ne tardera pas à se libérer, probablement grâce aux contacts avec les Portugais qui, discrètement, commerçaient avec Ngoyo et ne voulaient pas céder le terrain aux Hollandais qui convoitaient le port de Cabinda, considéré comme le meilleur au nord du fleuve. L'intérêt commercial des Portugais se portait alors sur le sel, le cuivre, l'ivoire, le poil de la queue de l'éléphant, le bois et surtout les tissus en raphia dont certains étaient utilisés comme monnaies (panos sambos pour les Portugais, mbondo fula chez les Woyo) et dont une grande partie était fabriquée exclusivement à Ngoyo $^{42}$.

\section{Emancipation de Ngoyo}

Vers 1606, les états de Loango, Kakongo et Ngoyo sont en relation commerciale avec le marchand Jorge Rondrigues da Costa à qui le Portugal avait loué l'île de Sao Ihome ${ }^{43}$. Ceci prouve l'existence à cette époque d'un état de Ngoyo autonome. Néanmoins, Ngoyo garda des liens de vassalité et nous savons par Van den Broeke, qui arriva vers 1612 à Mbanza Ngoyo, qu'il persista des rivalités politiques entre Ngoyo et Loango. Reçu par le Mangoyo, il rapporte ce qui suit: "Le roi est très vieux, c'est un homme cruel. Il est continuellement en guerre contre les insicus. Il est grand ennemi du roi de Loango" ${ }^{44}$. Tenant compte de ces éléments, particulièrement de l'âge accordé au roi ${ }^{45}$, on peut donc avancer que l'émancipation de Ngoyo est antérieure à 1600 . Par ailleurs, bien que la fondation du royaume ne fut pas une oeuvre de conquête, elle ne fut pas non plus pacifique ; il fallut se défendre contre des envahisseurs (que certains identifient aux Nsundii ou aux Babwende). Enfin en ce début du royaume, il est probable que Ngoyo ait plus lutté contre Loango ${ }^{46}$ que contre Kakongo ${ }^{47}$.

La suite du récit du navigateur hollandais contient d'autres renseignements intéressants : à savoir que Ngoyo a atteint sa dimension maximale, notamment à sa frontière sud constituée par le fleuve ${ }^{48}$ et que le pays est prospère. Quant à sa capitale, elle est située à l'intérieur du pays, loin de la côte. 


\section{L'organisation du pouvoir au Ngoyo}

sculptés ou non sculptés (zimpunji), un couteau de parade (cimpaba), une couronne (soku), un bâton de commandement ou sceptre (kooko la nzinga, "bâton de la vie") des couvre-chef divers (mpu) en peau de léopard, des manilles (soongo) et des bracelets (n'Iunga), des petits (kula) ou des grands (ngoma) tambours (zindungu) et des doubles gongs (ngongye). Dès lors, il devait observer quelques interdits :

- il ne pouvait plus se rendre au bord de la mer ;

- il ne pouvait plus porter des habits ou utiliser des ustensiles d'origine européenne ;

- il ne pouvait plus se trouver en présence d'une personne de race blanche.

41

La structure du pouvoir au Ngoyo s'apparente à celle de ses voisins Kakongo et Loango, bien que ce dernier évoluera d'une monarchie héréditaire vers une monarchie élective à la fin du $18^{\mathrm{e}}$ siècle.

Mangoyo, le propriétaire de Ngoyo ; son nom est précédé de la particule honorifique Mwe, Seigneur de (on trouve aussi Mwene et Ma). Devant être issu d'un couple princier, il s'en suit que d'étroites relations de parenté liaient les monarques et les dignitaires des royaumes côtiers ${ }^{49}$. Il est cependant bien connu que Ngoyo fut le principal fournisseur en femmes des autres royaumes. Ce qui lui donnait l'avantage d'être au courant de toutes les sources de conflit et par la position familiale de ses rois, d'avoir un rôle d'arbitre. gouverneur de la province côtière du royaume avec le titre de Mambuku et résidait non loin du port de Kabinda. Ce qui faisait de lui un homme riche et puisssant, souvent en rivalité avec le roi.

kaanda $)^{50}$, qui occupaient en fait le rang de neveux (mwana fumu) ou de petit-neveux (ntekele-fumu). Cependant, le rapport des forces entre les clans fut déterminant ; ce qui donna souvent lieu à de longues luttes internes et à des inter-règnes interminables.

jours, le futur roi était initié au savoir traditionnel et ésotérique et à l'art de gouverner ; il subissait les épreuves devant confirmer sa maturité humaine et spirituelle. Il recevait la consécration des prêtres (ntoma za si de certains esprits de Terre), Luunga à Lende, Lusunji à Ichizu, et Njimbi à Mbanza Ngoyo. A sa sortie de l'initiation, il parcourait le pays et procédait à des magnificiences ${ }^{51}$.

De retour à la capitale, il procédait aux obsèques de son prédécesseur dont le corps boucané était enterré au cimetière royal de Ngoyo.

e Mangoyo était entouré d'une cour de dignitaires qui au cours des siècles devint pléthorique. Il dirigeait le pays grâce à un gouvernement, un conseil des chefs de clans et des gouverneurs de provinces relevant de son autorité directe. Les principaux membres de son gouvernement sont :

- Makaya : en tant que premier conseiller du roi, il exerçait la fonction de premier ministre. Choisi en dehors de la noblesse, il devait souvent défendre son autorité contre l'ambitieuse et envahissante personnalité du mambuku qui, comme il a été dit, est proche parent du roi et son successeur présumé.

Civilisations, 41 | 1993 
- Mangovo za Ngoyo : un personnage issu de la noblesse chargé des affaires étrangères et des relations avec les étrangers. Il était aidé de mamputu qui avait la charge spécifique des relations avec les Européens.

- Mafuku : responsable des activités commerciales du royaume. Il fixait les prix, contrôlait la qualité des marchandises, assurait la sécurité des biens et des personnes dans les marchés et les ports. Il récoltait les taxes et les impôts du royaume. Il n'était pas toujours issu de la noblesse mais était souvent choisi dans la riche classe des courtiers. Sa fonction faisait de lui l'allié privilégié de la classe dirigeante.

- Mwelele : messager spécial de la cour, chargé d'annoncer la mort du roi et la désignation de son successeur. Avec deux autres dignitaires portant les titres de Mamboma et de Mangovo, Mwelele faisait partie du comité de régence devant assurer le pouvoir dès le lendemain de la mort du roi jusqu'à l'intronisation du nouveau monarque.

- Makimba : chargé de la production agricole, de la chasse et de la pêche.

- Plusieurs autres dignitaires relevaient des services directs du roi : Mambanza, chargé des finances du roi, alimentées par les impôts et les taxes sur les particuliers ; Mambele, messager du roi, il était reconnaissable par le couteau (mbele) d'apparat frappé aux emblèmes royaux dont il était porteur.

- Il existait enfin beaucoup d'autres charges dont plusieurs avaient trait aux pratiques religieuses dans le pays et à la cour.

\section{Conclusion}

L'émergence du petit royaume de Ngoyo n'apparaît que vers la fin du $16^{\mathrm{e}}$ siècle, un siècle après l'arrivée des Portugais sur les rives du fleuve Zaïre. Cette longue absence sur la scène politique de la région signifiait que le royaume était probablement encore dépendant des royaumes de Kongo et plus tard de Loango. Avec ce dernier, des liens divers vont persister notamment sous forme de tribut, en biens ou en femmes, payés à l'avènement ou à la mort du Maluango.

La formation du royaume de Ngoyo fut rendue possible grâce à la centralisation du pouvoir, jusque là détenu par les chefs de terre (fumu za si), les chefs de clans (fumu za makanda) et les chefs religieux (fumu mpezo). L'autorité a été remise entre les mains du roi, assisté des représentants de certaines catégories sociales. Assumant ces divers aspects de l'autorité, le roi est la clé de voute d'une architecture sociale délicate. Il en sera aussi le tendon d'Achille en ne laissant en place que le système de parenté comme seule structure sociale.

L'autre facteur qui favorisa la formation du royaume de Ngoyo fut la présence européenne à la Côte Atlantique. Celle-ci favorisa la création du port de Cabinda ainsi que le développement du commerce entre la côte et les régions éloignées de l'intérieur. $\mathrm{Ce}$ commerce exigeait une main-d'oeuvre abondante, notamment pour le transport de produits comme le sel et les biens européens qui devaient être échangés contre l'ivoire, le cuivre etc. Ce qui petit à petit transforma les relations entre les commerçants européens et leurs fournisseurs africains; car, petit à petit, les produits demandés vinrent à manquer. Il fallut donc chercher les principaux produits comme l'ivoire de plus en plus loin. D'autre part, des esclaves étaient de plus en plus demandés, et il fut nécessaire de s'en procurer ailleurs. Ainsi, les fournisseurs Woyo devinrent de plus en plus des intermédiaires, dans un commerce qu'ils ne contrôlaient plus totalement. Dès lors, le sort 
du royaume de Ngoyo fut lié à l'essor et au déclin de la traite transatlantique. Il ne put jamais s'en dépêtrer.

\section{BIBLIOGRAPHIE}

BASTIAN A. : 1874, Die deutsche expedition en Loango kuste, Costenoble, Iena, 2 volumes.

BATTEL A. : 1901, The strange adventure of Andrew Battel in Angola and adjoing Regions, Ravenstein, London.

BITTREMIEUX L. : 1936, La secte des Bakhimba au Mayombe, mémoire I.R.C.B., Bruxelles.

BOUET-WILlAUME E. : 1848, Commerce et traite des Noirs aux Côtes Occidentales d'Afrique, Imprimerie Nationale, Paris.

CRINE-MAVAR B. : 1984, Contribution à l'étude de l'histoire de Banana, Zaïre Afrique, nº 190, Kinshasa.

CUVELIER J. : 1953, Relations sur le Congo du Père Laurent de Lucques (1700-1707), Bruxelles.

Idem, 1955, L'ancien Congo d'après P. Van den Broeke (1608-1612), Bulletin ARSC, Bruxelles.

CUVELIER-JADIN : 1954, L'ancien Congo d'après les archives romaines (1518-1640), Bruxelles.

DAPPER O. : 1686, Description de l'Afrique, Wolfang, Waesberge, Boom et Van Someren, Amsterdam.

DEGRANDPRE L. : 1801, Voyage à la Côte Occidentale d'Afrique fait dans les années 1786-1787, Dentu,

Paris, 2 volumes.

DEMATTOS e SILVA J. : 1904, Contribuiçâo para o estudio de regiâo de Cabinda, Typografia Colonial, Lisboa.

GONCALVES A.C. : 1985, Le lignage contre l'Etat, dynamique politique Kongo du XVIème au XVIIème siècle, Instituto de Investigaçâo cientifica Tropica, Universidade de Evora.

HAGENBUCHER-SACRIPANTI F. : 1973, Les fondements spirituels du pouvoir au royaume de Loango, mémoire ORSTOM, Paris.

LABAT (Cavazzi), 1732, Relation historique de l'Ethiopie occidentale, JP Despine, Paris.

MARTIN Ph. M. : 1970 , "The trade of Loango in the $17^{\text {th }}$ and $18^{\text {th }}$ centuries", in Gray and Birmingham, eds: Precolonial African Trade, Oxford University Press, London, New York, Nairobi.

MARTIN Ph. M. : 1972, The external trade of Loango Coast, 1576-1870 (The effects of changing commercial relation on the Vili kingdom of Loango), Oxford University Press, Studies in African Affairs, Oxford.

MARTINS I. : 1956, Monarchia do Ngoyo,Portugal em Africa, vol. XIII.

MEROLLA J. : 1702, A voyage to Congo and several other countries, chietfy in Southern Africk, in A collection of voyages and travels compiled by A. Churchill, London.

MONTEIRO J.J. : 1875, Angola and the River Congo, Macmillan and C, London, 2 volumes.

MULINDA H.B. : 1985, La Société Woyo : Structures sociales et religieuses, thèse de doctorat réalisée sous la direction du Pr. Luc de Heusch, U.L.B.

NGUVULU A. : 1971, L'Humanisme négro-africain face au développement, Okapi, Kinshasa. 
NOGUEIRA J.A. : 1953, "Os povos de margem derita do Zaïre interior no terceiro quartel de seculo XVII", Boletimde Instituto de Angola, 1.

OLGIBY J. : 1670, Africa, Th. Johnson, London.

PIGATERRA Ph. et LOPES : 1963, Description du Royaume du Congo et des contrées environnantes (1691) trad. et annotée par Bal W., Nauwelaerts, Louvain, Paris.

PIRENNE J. : 1959, "Les éléments fondamentaux de l'ancienne structure territoriale et politique du Bas-Congo", Bulletin de l'ARSC, V, 3.

PULCHAS S. : 1613, Pulchas, his pilgrims, or relation of the world and religions observed in ail ages and places discorved from the creation unto this present, London.

PREVOST : 1776, Histoire Générale des voyages, P. de Hondt, La Haye.

PROYART : 1776, Histoire de Loango, Kakongo et autres royaumes d'Afrique.

SERRANO G.H.M. : 1983, Os senhores da terra e os homens do mar, antropologia politica de un reino africano, FFLC, Universidad de Sao Paolo.

VANSINA J. : 1990, Paths in the Kainforests. Towards a History of political tradition of Equatorial Africa, The University of Wisconsin Press.

VAZ J.M. : 1970, No mundo dos Cabinda, Estudio etnografica, L.I.A.M., Lisboa, 2 vol.

VOLAVKA Z. : 1970, Le Ndunga, un masque, une danse, une institution sociale au Ngoyo, Arts d'Afrique Noire, 1.

\section{NOTES}

1. NGUVULU A : L'humanisme négro africain au développement, Okapi, Kinshasa, 1971, p. 25.

2. Communication personnelle de Mr. Kanimba qui a effectué des fouilles à la localité Ngoyo (Zaïre) en 1987.

3. VANSINA J. : Paths in the Rainforests, Toward a History of Political tradition of Equatorial Africa.The University of Wisconsin Press, 1990, pp. 49-56.

4. HAGENBUCHER-SACRIPANTI P.: Les fondements spirituels du pouvoir au royaume de Loango, ORSTOM, Mémoire $\mathrm{n}^{\circ}$ 6, Paris 1973, pp. 22-25.

5. SERRANO, CMH : Os Senhores de Terra e os Homens do mar: antropologia politica de um reino africano, FFLCM-OSP Sao Paolo 1983, pp. 72-84.

6. MULINDA Habi B., La société Woyo : structures sociales et religieuses, vol. I, thèse de doctorat en sciences sociales, ULB, 1985, p. 222.

7. DE MATTOS e SILVA, J., Contribuicâo para o esrudio da regiâo de Cabinda, Lisboa, 1904, p. 301.

8. DAPPER O., Description d'Afrique, Wolfgang, Waesberge, Boon et Van Someren, Amsterdam, 1686, p. 340.

9. DEGRANDPRE, L., Voyage à la côte occidentale de l'Afrique fait dans les années 1786-1787, Dentu, Paris 1801, vol. 1, p. 167.

PROYART : Histoire de Loango, Kakongo et autres royaumes d'Afrique, Beston, Paris, 1/6, pp. 1-2.

10. DAPPER cité par MARTIN PH. M : The external trade of Loango, 1576-1870, Oxford Univ. Press, Studies in African Affairs, Oxford, 1972, p. 32.

11. OLGIBY J. : Africa, Th. Johnson, London 16/0, p. 521.

PREVOST : Histoire générale des voyages, de Hondt, Paris, 1757, pp. 164-185.

12. MEROLLA do SORENTO J., A voyage to Congo and several other Countries; in Churchill : collection of voyages and travels, London, 1702, p. 712. 
13. CUVELIER J. Relations sur le Congo du Père Laurent de Lucques (1700-1717), Bruxelles, 1953, p. 6.

14. BOUET-WILLAUMEZ E., Commerce et traite des Noirs aux côtes occidentales d'Afrique, Imprimerie Nationale, Paris 1848, p. 165.

15. MONTEIRO J.J., Angola and the River Congo, Macmillan and Co, London 1875, p. 254.

16. PULCHAS Samuel, Pulchas his Pilgrims, London 1613, Liv. VII, Cap. 346, p. 9/9.

17. Punto de Palmeino: nom qui fut longtemps donné à la Pointe de Banana (Jeune Palmier). CRINE-MAVOR, B. "Contribution à l'étude de l'histoire de Banana", Zaïre Afrique, n 190, 1984, p. 622.

18. OGILBY, op.cit., p. 521.

19. DAPPER, op.cit., p. 340.

20. MEROLLA, op.cit., p. 716.

21. PROYANT, op.cit., p. 132.

22. Bien qu'il soit probable que les frontières orientales de Ngoyo aient pu atteindre Boma, nous pensons que les dimensions que lui donne J. Pirenne sont quelque peu exagérées et ne correspondent pas à la réalité historique. PIRENNE J., "Les éléments fondamentaux de l'ancienne structure territorial en politique du Bas-Congo". Bul. Acad. Roy. Sciences Coloniales, 1959, V, S, pp. 557-577.

23. Deux versions similaires sont données par: MARTINS L, "Monarchia do Ngoio Portugal in Africa", Vol XII, 1956, p. 204 et SERRANO C.M.H. : op.cit., pp. 35-37.

24. Vumu et Fumba sont deux localités de la collectivité de la Mer au Zaïre. Vumu, dont il sera aussi question plus loin, est un salinage près de la mer. Le nom serait une onomatopée. Ce serait en effet le bruit produit lorsqu'on secoue dans un panier une quantité de sel pour le débarrasser de l'eau.

25. NGUVULU A., op.cit., pp. 39-40. L'auteur est un descendant d'un des clans royaux, établi à Siafumu au Zaïre. Par ailleurs, le même récit nous a été rapporté par le prêtre de Buunzi à Muanda.

26. Cette structure serait à la base de l'organisation politique du Ngoyo. A certains endroits les différents pouvoirs sont assurés par la même personne.

27. LABAT J.B. (Cavazzi) : Relation historique de l'Ethiopie Occidentale, J.B. Delespine, Paris, 1732, Vol. 1, p. 240.

BASTIAN, A., Die deutsche expedition en der Loango kuste, Costenoble lena, 1874, pp. 85-87 et 223-225, Vol. 11, p. 1/1.

BITTREMIEUX, La secte des Bakhimba au Mayombe, mémoire I.R.S.B., Bruxelles, 1936, pp. 135-137.

28. BASTIAN, op.cit., pp. 224-225.

DE MATTOS e SILVA, op.cit., p. 30.

29. SERRANO, op.cit., pp. 56-59.

30. Comme le souligne Gonçalves, dans les royaumes de culture kongo, le politique et le social constitue un enchevêtrement très serré comme les fils de chaîne et les fils de trame d'un tissu. Néanmoins, c'est le social qui assure la cohérence. C'est pourquoi on constate qu'aux disparitions successives des différents royaumes kongo, le social assure la continuité de la société alors que la politique est réduite à sa plus simple expression : la chefferie ou le village. GONCALVES A. : Le lignage contre l'Etat, dynamique politique Kongo du XVI ème au XVIII ${ }^{e ̀ m e}$ siècle, Instituto de Investigaças Cientifica Tropical, Universidade de Evora, 1985, pp. 223-225.

31. Nkhazi signifie défenseur (couramment traduit par oncle).

32. Seuls quelques vieux se rappellent encore cette institution. Le Bingu recommandait certaines formes de mariages notamment le mariage avec la CC ou avec une esclave. Dans le premier cas, les biens acquis restent sous la même autorité clanique et dans le deuxième cas, les enfants issus de ce mariage sont intégrés au clan du Père.

33. SERRANO, op.cit., pp. 72-84. 
34. VAZ, J.M., No Mundo dos Cabinda, Estudis ethnografico, ed. A.M. Lisboa 1970, Vol. I, pp. 41-42, Vol. 11, p. 66 et p. 155.

35. Bakama (sing. nkama) est le terme de respect qui signifie femmes; pour les masques, il caractérise le fait que les membres de cette association doivent obéissance aveugle aux prêtres du nkisi si. Par contre ceux-ci étaient sous le contrôle d'un conseil royal. Par ailleurs, on peut considérer l'institution des masques comme une vieille institution qu'ont connu tous les groupes de culture Kongo même si certains l'ont aujourd'hui perdue. Voir PREVOST, Histoire Générale des Voyages, tome sixième, nouvelle édition, Pierre D'hondt, La Haye, 1/5, p. $2 / 9$.

36. BASTIAN, A., op.cit., pp. 80-84.

VOLVAKA, Z., Le Ndunga, un masque, une danse, une institution sociale au Ngoyo, Arts d'Afrique Noire, 1, $19 / 6$.

37. Comme pour le bingu, il s'agit d'un mariage prescrit.

38. L'hypothèse de la création de Ngoyo grâce à un riche Portugais rapportée par quelques acteurs est assez révélateur à ce sujet. MEROLLA, op.cit., p. 716.

39. CUVELIER-JADIN, L'ancien Congo d'après les archives romaines (1518-1640), Bruxelles, 1954, pp. 161-162 et 195.

40. PIGIFETTA PH. et LOPES : Description du royaume de Congo et des contrées environnantes (1591) traduit et annotée par BAL W, Nauwelaerts, Louvain, Paris, 1963, p. 31.

41. BATTEL, A., The strange adventures of A. Battel in Angola and Adjoing Regions, Ravenstein, London, 1901, p. 42.

BRASSIO A.D. Monumenta missionaria Africain, Vol. V, 1955, p. 241 et suites avance la date de 1607.

42. MARTIN PH. "The trade of Loango in the $17^{\text {th }}$ and $18^{\text {th }}$ centuries", in GRAY and O. BIRMINGHAN (ed) Precolonial African Trade, Oxford Univ. Press, London NM Nairobi, 1970, p. 142. Le commerce d'esclaves ne portait alors que sur quelques milliers d'individus par an.

43. NOGUEIRA J.A., "Os povos da margem direita do Zaïre interior no terceiro quartel de século XVII", Boletim in do Instituto de Angola, I, 1953, p. 19.

44. CUVELIER, L'ancien Congo d'après P. Van den Broeke (1608-1612), Bull. ARSC, Vol. I, $\mathrm{n}^{\circ} 2,1955$, p. 190.

45. Contrairement à ce que dit Ivaristo Martins qui la situe en 1690. MARTINS I. : "Monarqui a do Ngolo", Portugal em Africa, vol. XIII, 1956, p. 204.

46. On trouve les traces de la présence de Longo dans les expressions comme "si Mweloango", la terre de Maloango pour parler du pays tout entier. On trouve aussi dans certains rituels d'investiture l'expression "besi Kongo, habitants de Kongo" pour désigner les Woyo, généralement d'ailleurs pour contester le pouvoir à quelqu'un.

47. Les conflits avec Kakongo débuteront ultérieurement sous l'instigation de Soyo.

48. Le voyageur l'atteint et le traverse le 12 mai 1612. C'est à la suite du conflit entre Ngoyo et Soyo en 1631 que la frontière fut ramenée à Mamputu.

49. Du temps de Degrandpré, le Mongoyo était le fils d'un dignitaire d'un royaume de Kakongo.

50. Sambo Mankata-Kalombo, Simbo et Mpuna d'après SERRANO, op.cit., p. 58. Certains de ces clans résultent des migrations successives (surtout au 18ème siècle).

51. A cause des dépenses occasionnées par l'intronisation ainsi que l'obligation de résider loin des ports commerciaux, les deux derniers monarques ne furent pas intronisés et leurs successions refusèrent de régner. 


\section{RÉSUMÉS}

The kingdom of Ngoyo emerged towards the end of the 16th century, a century after the arrival of the Portuguese on the banks of the river Zaire. The formation of the kingdom was made possible by the centralisation of power, authority being in the hands of the king helped by representatives from certain social categories.

The other factor involved in the forming of the kingdom was the European presence on the Atlantic coast. This presence helped the creation of the port of Cabinda and the development of commerce between the coast and the interior regions far from the coast. This commerce needed a considerable amount of manpower for the transport of salt and European goods which were exchanged for ivory and copper. Gradually these products became in short supply and it was necessary to seek both manpower and ivory further and further away. From this time, the fate of the kingdom of Ngoyo was linked to the growth and decline of the transatlantic slave trade.

\section{AUTEUR}

\section{HABI BUGANZA MULINDA}

Institut des Musées Nationaux du Zaïre - Kinshasa - Zaïre 